fact that our specimens show new formed blood-vessels in the inflammatory tissue within the vitreous of certain of these eyeballs lends some support to such a contention. The conditions are, however, not quite parallel, the lymph channels in the cornea being, under normal conditions, more definite than those in the vitreous, where the process of nutrition is conducted by osmosis rather than by lymph flow. The vascularization of the cornea takes place much more readily in its more highly organized structure than does the same process in the vitreous. In the end, however, the difference would appear to be a matter of degree rather than of kind.

So long as those who use the terms understand exactly what they mean, little harm can be done, but, isasmuch as those terms will be transmitted to students and to many others, whose comprehension of the position will not be clear, it seems a pity to use the term "hyalitis."

The appearances so constantly observed in the vitreous, taken together with those found in several of the optic nerves, would seem to lend strong support to the view that some, at least, of the lymph from the eye takes a backward passage and escapes by channels in, or in the neighbourhood of, the optic nerve. This phenomenon serves to explain the occurrence of optic neuritis as a complication of septic wounds of the anterior segment of the globe.

\title{
FACTORS IN STEREOSCOPIC VISION AND IN THE VISUAL ESTIMATION OF DISTANCE
}

\author{
BY \\ E. M. EAton, M.D., \\ CAPTAIN R.A.M.C.
}

THE power of estimating distances with accuracy is essential to expertness in handicraft, in sport, and in any activity which controls the direction or the amount of a mechanical action.

In every department of life, therefore, this faculty is of importance, directly or indirectly; yet, owing to its very general possession and to its susceptibility of improvement according to the requirements we habitually make of it, the limitations and the illusions to which it is subject are readily overlooked. In few judgments are we more dependent on familiarity of circumstances and setting than in this one. The question is one which has assumed a peculiar importance in recent years, when so many men accustomed to living indoors have been transferred to a life in which visual judgments in the open are constantly called for, 
and when for the first time the art of flying has become an achievement of far-reaching consequence.

Stereoscopic vision-literally solid vision-has since the time of Wheatstone been commonly accepted as a specific function of the visual apparatus whereby we appreciate solidity in space as a result of fusion of the dissimilar images formed on the two retinae. This view received much of the stability of its acceptance through the writings of Hering, who regarded stereoscopic vision as a congenital property of the visuo-psychic apparatus.

While the nativistic theory has never been widely accepted, the general view of the essential nature of solid vision is seldom seriously questioned. On the other hand, the belief that a single eye gives a certain amount of appreciation of three dimensions, to the degree, at any rate, which is often referred to as the "sense of depth," is surely general;, and the opinion which I shall advocate that this ought rightly to be included in the term "stereoscopic vision" is not new; it is implied in the existence of such instruments as uniocular stereoscopes and binocular stereoscopes for use with a single picture.

So far as I am able to conceive a difference between the "sense of depth" and the "sense of solidity," it is merely this, that the former is more realistic in reference to the intervals between objects, and the latter in reference to the objects themselves. Even if such a differentiation did tend to occur, however, the composition of most views demands that if the one is observed so also is the other. In no type of vision, except in specially arranged illusions, do we see the further parts of an object detached from the nearer ones, a state which would follow if we perceived depth without solidity. The difference is one of sentiment rather than of perception; the sense of depth gives a wet atmosphere picture, the sense of solidity a bright one.

There are other means than the fusion sense* whereby we perceive solidity through vision. Apart from fusion, I would include five factors as being of the first importance, and generally available. The chief of these is the shape of the retinal images of symmetrical objects or of objects of whose real shape we have some other means of gauging; what, in fact, is generally understood by the term "sense of perspective." There is alteration of definition according to distance; there are the lighting effects; in the case of very near objects there is the sense of ocular convergence; and, if the

\footnotetext{
*In order to avoid ambiguity, I shall use the term "fusion sense" in place of " stereoscopic vision," to denote the specific contribution of binocular vision to the sense of depth or solidity; it will, therefore, not be interchangeable with the term "fusion faculty." which describes a function that is antecedent to the fusion sense thus defined. "Stereoscopic vision" will be used in the less restricted and less conventional, but, I think, not less legitimate, meaning of the visual sense of solidity, however engendered.
} 
observer is moving or should move his head, there is parallax. There are also others of lesser value and more occasional occurrence, such as atmospheric interference; and many incidental evidences, as, for example, the manner in which a cloud of steam may be broken as it passes through a group of trees, thus giving information concerning the relative position of the obstructions. In addition there are several important accessories, but notably the muscle sense, which, in so far as it acts as an informant of the position of the eyes relatively to the body, or of the distance through which a head movement has been made for the observation of parallax, ought to be grouped with the sense of convergence as an extension of the sense of vision.

Although these factors assist in the recognition of depth, it would none the less be unjustifiable to describe them as means of stereoscopic vision if they gave us merely knowledge of solidity, but failed to give us that quality of visual feeling by which from sensation alone, without conscious reference to intellectual knowledge, we become aware of the third dimension. If, however, they or some of them should give us this quality of consciousness, I think it illogical to reserve the term "stereoscopic vision" for the vision obtainable by any one particular means. It is objectionable on other grounds also ; it may lead us into fallacious deductions. The sense of depth obtainable from fusion is an art acquired by experience no less than is the sense of perspective, and it is a much less fixed character of our visual functions. If the same art can be acquired without the aid of fusion there is no reason to discriminate.

To my mind a one-eyed man sees stereoscopically in the only valid sense of that word. He will always say that he sees things solid, not flat, and his achievements support his belief. It is a common experience that a woman who has recently lost the sight of an eye will pour tea from a pot upon the table cloth beyond the cup that she intends to receive it; but after a little while she becomes perfectly efficient in the matter. She learns to pay more attention to, and to place more reliance upon, the other checks of the accuracy of her solid vision.

If we look at an ordinary pair of stereoscopic photographs and, without removing the instrument, cover one eye, we shall find that we still see stereoscopically. There is a certain loss undoubtedly, we lose half our illumination, and we may feel that solid vision is not of so great a realism as it is with the two eyes.* This improves with practice and effort, however; in such an experiment we are in the position of the woman, of whom I spoke, on the very day on which she loses the sight of her eye. If we look at the people and objects

- This loss is much less if an optically correct stereoscope is used. The stereoscopes ordinarily sold are correct neither in focal length nor in prismatic strength, and the photographs sold with' them are made with an exaggerated distance between the lenses. 
around us, and then cover one eye, the view does not become flat. It is true we lose in the accuracy of relative judgment of distance, but we do not lose the sense of solidity; in fact, if synchronously with the covering of one eye we bring the other eye into the position formerly occupied by the middle of the interocular space, so that the line of projection will not be altered, we shall have difficulty to discover any modification of the visual impression. The fusion sense is not an essential factor in stereoscopic consciousness, and I think I shall be able to show that it is not the dominating factor.

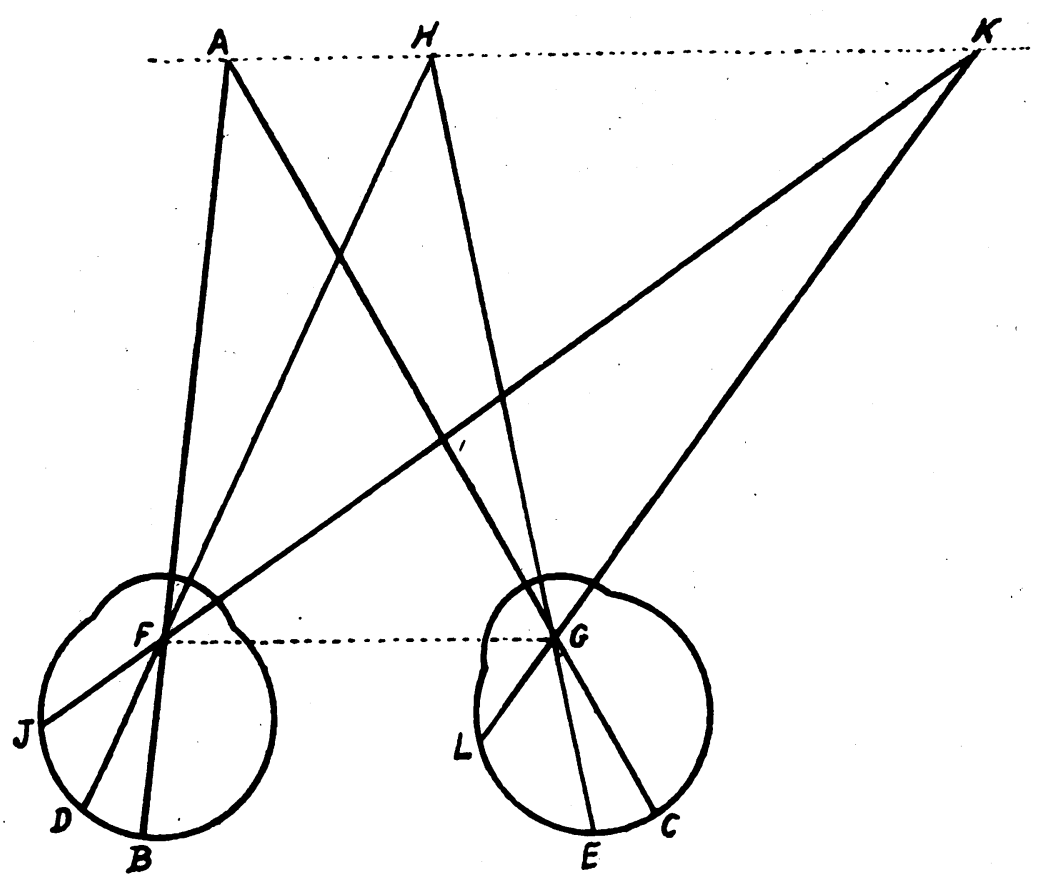

Fig. 1.

A glance at the theory of the fusion sense will indicate something of its potentialities and its limitations. The classical theory of corresponding retinal points is still useful, although in some respects it cannot be accepted rigidly. When the visual lines are parallel, that is, when vision is fixed on a distant object, light from the point of fixation will fall on the two foveal centres, and the rays from any other distant objects will fall on points of the two retinae, which form at the respective nodal points of the eyes, with the lines of vision, equal angles in parallel planes. Such points on the retinae are known as corresponding points. Now, if the eyes converge to fix a comparatively near object its images will fall on 
the maculae and the images of all other objects in the same plane would, according to the original theory, fall on corresponding points. The plane of the objects fixed was known as the reference plane or basic plane, for that particular position of the eyes.

To say that the images of points on the reference plane fall on corresponding points on the retinae is true, however, only for a very limited field, strictly true in fact only for distant objects; the images on the periphery of the binocular field are double even for one plane.

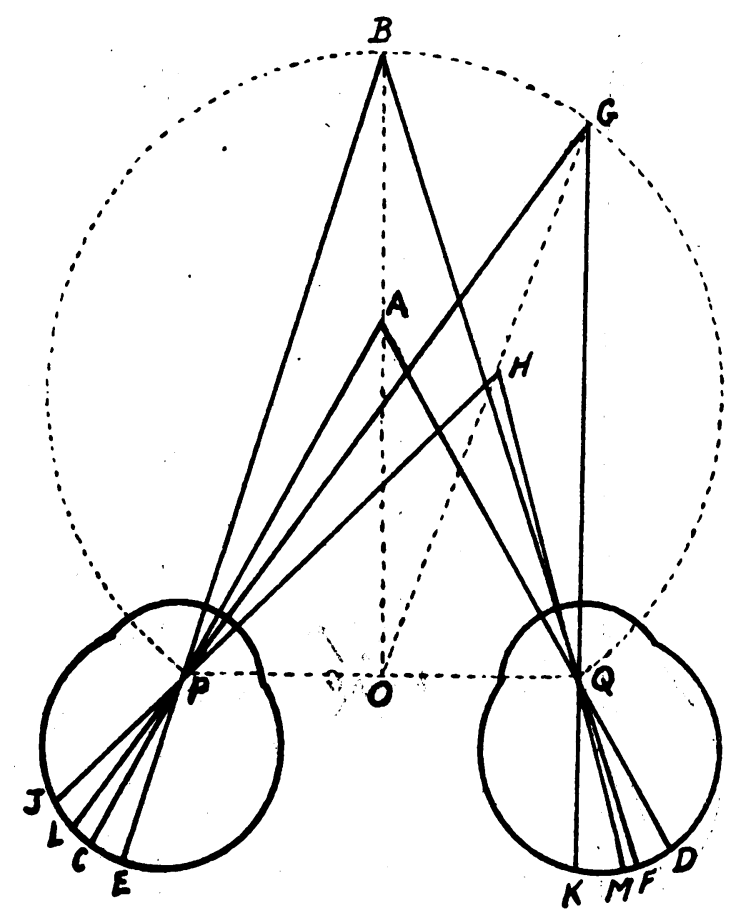

FIG. 2.

If in the accompanying Fig. $1 \mathrm{~A}$ is the point of fixation and $\mathrm{B}$ and $\mathrm{C}$ the two maculae, $\mathrm{D}$ and $\mathrm{E}$ will be corresponding points according to the definition if $\angle \mathrm{CGE}=\angle \mathrm{BFD}$. But $\angle \mathrm{CGE}$ will equal $\angle \mathrm{BFD}$ only if $\angle \mathrm{HGA}=\angle \mathrm{HFA}$. The further $\mathrm{H}$ is displaced laterally from $\mathrm{A}$, for example to the point $\mathrm{K}$, the less is this true. and if the binocular field reached out to $90^{\circ}$, which of course it does not, the difterence on the periphery of the field would equal $\angle \mathrm{GAF}$, which is twice the angle of convergence and is therefore a very large angle when we are looking at near objects. To overcome this difficulty, it is necessary to postulate that the surface of reference should be circular in. section; this circle which passes through the 
point of fixation and the nodal points of the two eyes, is known as the horopter; the successive reference surfaces, as the point of fixation approaches to the observer, will therefore be, in section, gradually diminishing circles which, obviously, cannot be concentric.

Continuing, however, if an object lies beyond the reference surface its images will fall to the nasal side of corresponding points, and if it lies nearer than that surface, they will fall to the temporal side. The amount of the dissociation will determine our estimate of the advancement or recession; it is really an unconscious

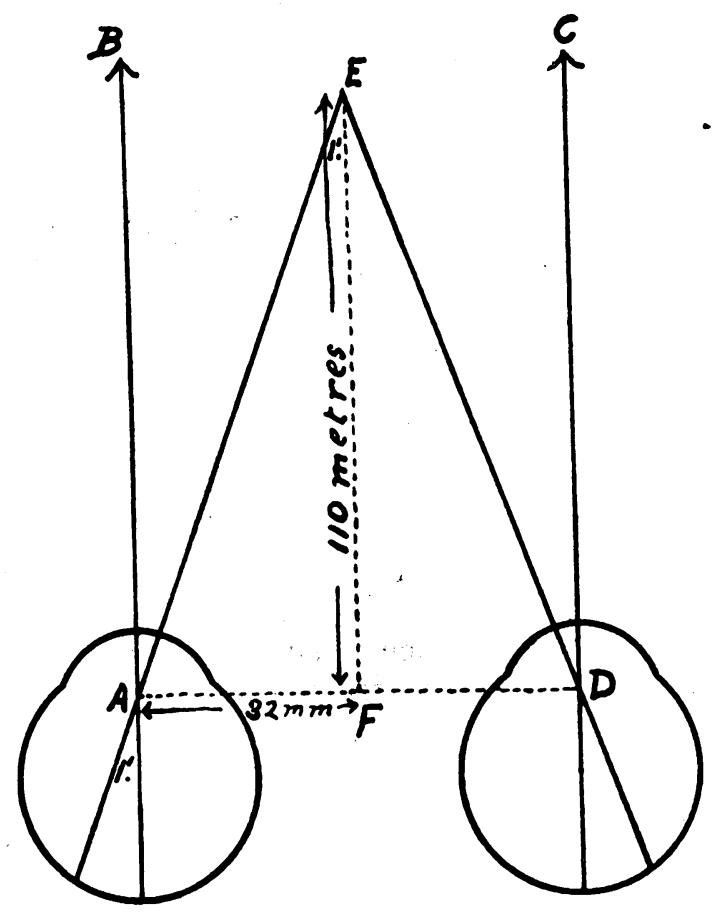

Fig. 3.

A B, D C. parallel lines of distant vision; F E, line of binocular projection; E, furthest point on this line giving appreciable angles at $\mathrm{A}$ and $\mathrm{D}$.

measurement of this angle that provides the means of our fusion sense judgment. The relative positions are shown in figure 2.

It will be observed that if the eyes fix the point $\mathrm{B}, \mathrm{E}$ and $\mathrm{F}$ being the maculae, the images of the point $\mathrm{A}$ fall to the temporal sides at $C$ and $D$. Light from any other point in the horopter of $B$, such as G, falls, as we have seen, on corresponding points, which let us call $\mathrm{K}$ and $\mathrm{L}$; again, light from any nearer point along the line $O \mathrm{G}$ will fall relatively towards the temporal sides, those of $\mathrm{H}$ falling at the points $\mathrm{J}$ and $\mathrm{M}$. 
Our power of measuring this angle of dissociation is of a very high degree, so that within the limits of distance that will aftord an angle appreciable by any means short of the finer types of mathematical instruments, our fusion sense will be eftective, provided, however, it is not interfered with by other influences. This is demonstrated in the old experiment of Hering's, of dropping beads on either side of a stretched thread, which is observed through a narrow funnel.

Visual acuteness of $6 / 6$ is such as will enable us to distinguish two points if the distance separating them subtends an angle of one minute. It is approximately the minimum angle which permits of the stimulation of two retinal cones leaving one cone unaftected between them. Assuming this standard of vision and an interocular distance of $64 \mathrm{~mm}$., an appreciable dissimilarity of images in the two eyes could occur, theoretically, up to a distance of 110 metres.

Beyond that plane the dissociation on the retina would not be such as to make differentiation anatomically possible. Our fusion sense, therefore, cannot play any part at distances greater than 120 yards, and in actuality it probably ceases to be effective at a considerably shorter distance; for points which do not fall on the maculae it necessarily fails at a much nearer plane. It is therefore of very limited range. It is also of very defective stability.

If a pair of stereoscopic photographs be reversed so that the lefthand card is seen before the right eye and vice versa, the view should be reversed in the third dimension, making objects in the foreground appear in the distance. This, however, will not be found to occur. It will sometimes be felt that a struggle is taking place, and a massive or obtrusive object in the foreground may sometimes momentarily move out of position, but in general the picture retains its original character. The other factors in stereoscopic vision dominate the fusion sense.

This experiment was referred to by Dr. I. Franklin in a paper published in the American Journal of Ophthalmology of April, 1918. Dr. Franklin's conclusion is that the picture is reversed, from which he argues the importance of seeing an object from two points of view, but he opposes the theory of stereoscopic effect being due to the fusion of dissimilar images as ordinarily accepted. The reversal to which he refers, however, must be the movement of a prominent object to which I have referred, for no ordinary composite view will be found to reverse against the evidence of the sense of perspective. When a massive object in the foreground moves to the back, it does not do so in accordance with any system of order, it is merely lifted out of the picture, that is it ceases to have any relation to the perspective projection of the view as a whole. This anomaly would less readily occur if the pictures were not made with an exaggerated displacement. Experimental pictures of symmetrical objects, such 
as cones and hemispheres, which are so arranged that the anterior and posterior perspective projections are acceptable, will, of course, reverse completely.

If we reverse the card top to bottom and right to left, that is by simple reversal without altering the original relationship of the two halves, the fusion-sense acts normally; but owing to the unusual view, the sense of perspective and the appreciation of shadows are almost wholly in abeyance. If now the two views are transposed, right to left and left to right, the fusion sense will have free play and immediately, being relieved of conflict, will carry the foreground into the distance, and distant objects will come forward. If there be a figure of a man shown in front of a building it will be found that there appears a clean cut hole in the wall exactly of the form of the man, and in the distance beyond will be seen the man himself. After some study the sense of perspective may begin to apprehend the inverted picture; if so it will again assert its dominance. The second part of this experiment has a particular value in eliminating the question of the assistance of intellectual knowledge which might be held to account in part for the maintenance of the normal relationships in the former method. In this case reversal occurs despite the fact that the observer is aware of the physical impossibility of the apparent relative positions.

Our fusion sense, therefore, appears to be extremely elastic and readily capable of compromise or even suppression; it works hand in hand with the other means of stereoscopic vision, giving and taking as may be most convenient on the occasion.

A painted picture does not appear to us flat, yet our fusion sense ought to make it so, for the two retinal images are similar. There is a feeble eftort in this direction, however, and unless we are accustomed to suppressing this, we shall get better relief with one eye. Our experience is that things that appear of the form we see in the picture are not flat, and our sense of perspective indicates the varying degree of relief. It is, of course, possible to look at the canvas and paint as such, and see a flat surface; but that is another matter. The $\epsilon$ ffect becomes more realistic if we examine an ordinary photograph in such a way as to get full value out of its perspective. Viewed in the usual way, the perspective of a photograph is truthful only in an objective sense. It is as though we were looking at a picture of a man viewing a landscape, and what is represented is what this man sees. We may project ourselves into the position of the man, and the perspective will be correct so far as intellectual considerations go; but in order to have his visual consciousness, we must be where he is, that is where the camera lens was; it is to this point that the lines of the picture converge, it is the centre of projection. The ordinary pictorial postcard is made as a rule with a lens of 5 inch focal length, and with the plate in the principal 
focal plane; it should, therefore, be held at 5 inches from the eye ; in that position we shall see it directly, we shall see it as though we ourselves were looking at the scene, not as though we saw someone else looking at it. We therefore see it in a real sense, there is no conscious mental process intervening between our visual sensations and the feeling of the presence of the objects depicted; in consequence we see stereoscopically. To focus for 5 inches we shall require a lens, and as we are going to believe ourselves looking at a distant scene, our accommodation should be relaxed. A $+8 \mathrm{D}$. lens

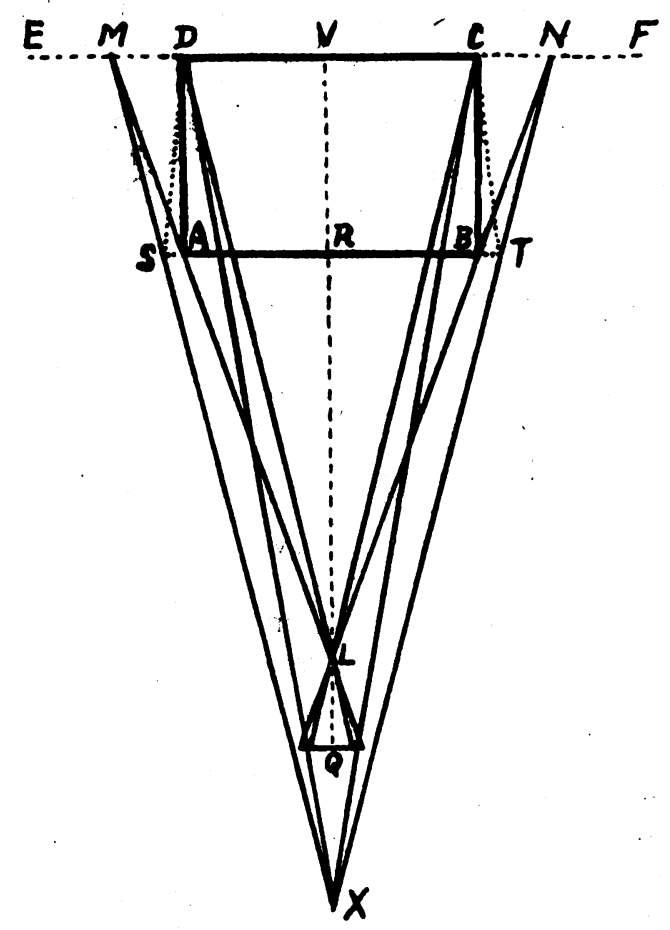

FIG. 4.

will therefore be required in addition to our distance correction if one is worn; the card should be held at the furthest position affording clear definition. Only one eye must be used, as it is essential that the point of observation be opposite the centre of the card.

The means whereby perspective gives accurately a sense of depth, and the reason why it is advantageous to view a photograph from the focal distance of the camera lens, will be made clear by the accompanying figure:

If $A B C D$ is a rectangular building of depth $R V, 20$ feet; $L$ a 
camera lens 40 feet in front of A B; E F an imaginary screen immediately behind the building; then $\mathrm{M} \mathrm{N}$ will represent an enlarged and re-erected image of that on the camera plate in the ratio $\mathrm{VL}: \mathrm{LQ}$. As viewed from $\mathrm{L}$ the image of the wall $\mathrm{DC}$ is not enlarged; but $\mathrm{A} \mathrm{B}$ appears as $\mathrm{M} \mathrm{N}$, enlarged in the ratio L V : L R, i.e., $60: 40$ or $1,5: 1$. Now $L$ is the only position from which the points $\mathrm{M}$ and $\mathrm{N}$ on the screen can be seen along the lines $\mathrm{L} \mathrm{M}$ and $\mathrm{L} \mathrm{N}$ on which the positions of $\mathrm{A}$ and $\mathrm{B}$ lie. If viewed from $\mathrm{X}$ the points $\mathrm{A}$ and $\mathrm{B}$ must be referred to positions on $\mathrm{X} M$ and $\mathrm{X} \mathrm{N}$, such as $\mathrm{S}$ and $\mathrm{T}$, and the building will no longer appeàr rectangular, but have the form S T C D. We know, however, that it is rectangular, for, while an individual building might have a freakish shape, the mind will not accept the suggestion that chimneys, windows, doorways, and all are built skew. Yet memory of form is insistent, and the reconciliation is found in our taking an objective view of the picture, including the observation point $\mathrm{L}$; we consequently lose the sensation of the extension of the scene in space before us. If we regard the camera as an eye looking at the building, the image $\mathrm{M} N$ will now represent an enlarged and re-erected image of that on the retina. The proportions $\mathrm{M} \mathrm{N}: \mathrm{D} \mathrm{C}$ being $1,5: 1$, determine that $\mathrm{L} \mathrm{V}: \mathrm{L} \mathrm{R}$ is also $1,5: 1$, an accurate interpretation of the stereoscopic relationship of $\mathrm{D} \mathrm{C}$ to $\mathrm{A} \mathrm{B}$.

If the objects of view be circles a greater accuracy of form judgment is required in making a correct interpretation, but it is still very easy. With the miscellaneous objects, such as trees, houses, or men, found in an average composite view, our sense of perspective remains the most important of the purely visual calls for stereoscopic consciousness, although not necessarily the most accurate informant for stereoscopic judgment.

Unlike the fusion sense, the sense of perspective has no natural limit to the distance within which it acts. Accuracy of perspective judgment is dependent on the form sense and the form memory. To use the same terminology as for light, the form sense here required might be described as the form difference as distinguished from the acuteness of vision which is the form minimum. The perception of form, and therefore also the sense of perspective, in a broad angular field involves not only visual impressions, but also impressions of ocular movements.

In judgment of distances we have instinctively a greater confidence in determining the relationship to imaginary straight lines drawn transversely in front of us, through the successive points of fixation, than in estimating relative radial distances, that is, positions in relation to concentric circles around us; least of all could we suggest a relationship to any horopter. This fact fits more easily into the scheme of a perspective estimate than of a fusion one; 
but, however that may be, the important point is, that in practice the reference surfaces for our composite stereoscopic vision are flat:

The next factor in stereoscopic vision is gradation of definition. If we look at a landscape with trees, houses, and other objects, we shall observe of the trees in the foreground the gnarled surface of their bark and the veined structure of their leaves, while of the houses we may discern the manner in which the stone is finished, and many other details. A little further off we can just discriminate separate leaves, and we still see the outlines of the blocks of stone in the house fronts. Further off still, the trees are represented only by their general outlines and by their colours.

An interesting light on the importance of the progressive definition of detail is given by its influence in stereoscopic radiography. This is the chief respect in which skiagraphy: fails to produce an optically correct representation of reality, and it proves a serious defect where the structure of the object is finely meshed. Unlike that of a photograph, the detail of a radiogram improves as the distance of the things represented increases, for the further parts are nearer to the plate. If a stereoscopic pair of radiograms be made, showing a hand with outspread fingers lying obliquely at about $70^{\circ}$ to the plane of the plates, it will probably be found quite impossible to get a stereoscopic impression, so persistently does the definition demand that the finger which is best defined be brought to the front of the picture.

In our estimates of distance when looking over a plain or over the open sea, definition is of the greatest importance; no index remains to our judgment but the texture of the undifferentiated carpet which stretches in front of us.

Gradation of definition as an aid in stereoscopic vision varies in effectiveness according to the acuteness of vision of the observer.

The sense of convergence is active at close distances only; but in the stereoscopic view of more distant objects, the determination of our relationship to near ones is essential. Of this I shall speak later. The great influence of convergence on our judgments at close range is observable in the use of the Wheatstone stereoscope. While keeping the photographs at fixed distance, if we move the mirror back and forward the effect is to make our eyes converge and diverge. Although the images subtend practically constant angles, they diminish as the mirror is pushed back, and appear larger when it is pulled forward, for our subconscious estimate of distance is altered ; the illusion is analogous to the apparent variation of the size of the moon, which, when it first rises and looks as if sitting on the horizon, seems larger than when it comes overhead and appears but a little above the clouds. The same experiment demonstrates our greater sensitiveness to convergence changes than to the absolute 
degree of convergence, as the illusion is more vivid while the movements are being made.

Lighting eftects afford very definite information, which is especially marked if the light is direct like that of the sun on a bright day, or of the moon or the artificial light by night. Where I sit when writing, if I close one eye, the shadows cast by some picture cords opposite compel me to recognize the distance of the cords from the wall. By expelling from the mind the association of the shadows with the cords, I can project the latter back to the wall, but until this is done the effect is undoubtedly and inevitably in relief. The varying density of the shadows in the folds of a heavy plain curtain at my side give a more accurate appreciation of the form of the curves than even binocular vision does on a part that is fully illuminated.

These are simple cases where the shadow effect is acting alone, but in more complex views shadows have generally an important part; it is noteworthy that in outdoor scenes weather relationships commonly determine that as we lose in shadow assistance, we gain in the value of atmospheric interference. In our interpretation of shadows we are to a large extent dependent on experience, more especially of the habitual interpretation of the effects of parallax ; for the rest, our sense of light difference is put to test.

The last of the major factors in stereoscopic vision which I shall mention is parallax itself. If with one eye closed and from a horizontal elevation we look at a number of plain opaque cylindrical vessels, such as earthenware flower vases, set out on a table, our stereoscopic guides will be almost entirely eliminated and our sensation of depth will be poor or absent. Our judgment, if not one of flatness, will, at best, be hopelessly fallacious. If, now, the head is moved laterally an immediate improvement occurs, and what may have been a flat view suddenly stands out in relief. The effect of parallax as presented to us whilst travelling by train is familiar; it would be hard by any effort of will, however complete might be the exclusion of other stereoscopic aids, to flatten out a view bearing such unmistakeable evidence of varying depth. Probably most persons have avoided the unpleasant sensation of this view to an extent which has prevented their acquiring it as a direct factor in stereoscopy; it still acts through a conscious interpretation. It might therefore be said that it is not legitimate to regard such a condition as an element in stereoscopic vision. This, however, may or may not be true.

In considering alterations of a state of consciousness brought about by external stimuli, it would surely be artificial to separate off such modifications of the resultant as arise from necessarily associated internal conditions. Were we to do so our vision would be meaningless. 
Thus it is that in stereoscopic vision, as in vision as a whole, previous knowledge is the greatest of all factors. Our strongest incentive to the introduction of a third dimension into a view is not directly visual, but is the belief that solidity exists in it, that it would be incongruous to believe it otherwise in the conditions observed. When we have once been convinced of the existence of the third dimension in a scene, the question whether we see stereoscopically is purely a psychic one; if the knowledge passes to subconsciousness and yet retains its influence on our visual concept, then we actually do see stereoscopically. The relationship is the same in the sphere of perception as consciously controlled action is to acquired reflex in the sphere of motor function. In each case the subconscious is the later development and the more reliable condition; in each case it is acquired by custom and correspondingly it may be improved by training.

Of the sensory factors which contribute to stereoscopic vision, the relative influence varies with persons and with circumstances. Not always does the one which most strongly compels the sensation of depth give the greatest accuracy in determining its amount. For average conditions I should place them in order: perspective, fusion sense, gradation of definition, convergence, lighting, and parallax. In special cases parallax would occupy a much higher place. Straub first claimed that he could see a swollen optic disc in relief after observation of its parallax. It is doubtless a common acquirement, although perhaps not always realized by those who possess it ; but it is a valuable means of measuring variations of the disc and is worth attention, physiological cups being good material for observation. In this case parallax is the chief guide, but perspective, definition, and lighting have also an influence.

My reasons for considering the fusion sense inferior to the sense of perspective are that it is more easily deceived, that its range of action is much more limited, and that acceptance of its guidance is much less imperative. The tests on which we are apt to appraise the value of binocular as compared with uniocular vision are experimental ones in which the index of dissimilar images is put to its greatest advantage, and the guides to the single eye are designedly excluded. As an indicator of the relative general value of the two functions it would be no more ludicrous to make an experiment of a converse type, and to compare the uniocular impression of a series of transversely stretched wires of which the lateral attachments were visible, with the binocular impression of the wires alone, as seen through an aperture in a screen. In everyday life our vision is rarely without perspective aids, but much of what we look at is beyond the range of dissimilar images.

In general it may be said that each function acts as a check on and reinforcement of the others where their ranges overlap, and as a 
complement where the limits of action of one or another fall short. All are capable of making compromise, the fusion sense and the sense of convergence most readily. Each factor acts to greatest advantage if the conditions in relation to it correspond to habitual experience.

Whilst stereoscopic vision involves the subconscious estimation of relative distance it does not necessarily follow that it implies any estimate of absolute distance. It is true, as we have seen, that every view has a definite relationship to the point of observation; the lines of perspective converge upon it, and if it is a short range binocular view the relationship could be calculated also from the dissimilarity of retinal images. Owing to the smallness of the angles, however, the subjective relationship determinable in this way is very inaccurate; while on the other hand, relative distances are appreciable in a high degree. But we are not dependent for the knowledge of our subjective relationship upon a single stereoscopic view. Only exceptionally is our vision fixed for more than an instant on one spot, and as fixation moves the reference surface changes. If we are approaching some definite point in space, as in drawing up a motor car at a door step, we have certainly a principal fixation point; but our relationship to it is changing and therefore also the horopter, and still more markedly all of our perspective ratios are continuously altering. Our conscious relationship to the external world is determined by the composite result of a potentially infinite series of stereoscopic pictures lying between the furthest and nearest planes for which we fix, together with a knowledge of our relationship to those fixation points which fall within a comparatively short range; therefore if we have no available foreground our distance estimates will be poorer.

How is the last link filled in between the nearer reference planes and our eyes? All our stereoscopic factors are at a greater advantage, it is true; but while that may assist I do not think it does so to any great extent. It is here that the muscle sense becomes of great importance. The ocular convergence comes into action, a function to the sensitiveness of which reference has already been made. If we should be standing on the level, the amount of depression of the visual lines in looking at an object on the ground, taken together with our own height, gives the data for the solution of a triangle, which custom reduces to a simple perception. Were we sitting in a motor car the radiator cap or the tip of the mud-guard would fix the position of the chief of our near hand reference planes; in making judgments from the bridge of a ship it would be the stem of the ship or some part of the rigging; such points of accustomed reference as these acquire an important value. In estimates of this kind everything that determines our sense of equilibrium and our knowledge of the 
position of the head and eyes also plays a part. The visual angle subtended by objects of known size is a factor of some importance, especially for objects lying in the peripheral parts of the field where most of our aids are at a disadvantage. The information obtainable from the act of accommodation, apart from convergence, is, on the other hand, practically negligible. It is possible to say which of two very near objects requires the more accommodation, but we cannot estimate the relative amounts.

It is noteworthy that in the majority of the practical applications of our estimate of distance we are concerned not with the naming of so many yards or inches, but in the judgment of the relationship of the effect of some proposed action to the space within which it is required to operate. If these judgments are carried out subconsciously the attention is free, as it should be, for the observation of variable factors alone; and, moreover, co-ordination is more rapid and less liable to confusion than if directed consciously.

The subconscious judgment of distance in this sense is but an attribute of the function of stereoscopic vision in the more extended meaning; when the eyes and mind are familiar with the circumstances amidst which for the time being they act, the judgment of distance becomes reduced to a simple sense perception.

The inferences that I would draw from these observations are:

1. That stereoscopic vision implies and, from its nature, is coextensive with the subconscious judgment of distance by vision.

2. That the potentiality of the individual for reliability in distance judgment must be gauged by considerations which include :-

1. Form sense.

a. Acuteness of vision.

$b$. Form judgment (form difference).

c. Form memory.

2. Fusion sense.

3. Convergence sensibility.

4. Light sense.

a. Light minimum.

b. Light difference.

c. Colour sense (in special cases),

5 Muscle sense.

6. Aptitude in visual observation,

3. That persons with defects which reduce the efficiency of the fusion sense, or throw it out of action, although deprived of a great asset in stereoscopic vision and in the judgment of distance, lose in degree only, and therefore may, by training, compensate in great part for the deficiency.

In discussing the nature of stereoscopic vision and the power of judgment of distance, I have not attempted to do more than 
indicate the elements of which I conceive these faculties to be made up, and to suggest the probable order of their influence. The assigning of relative values to the various factors and the determination of their modifications according to circumstances whether of environment or of ocular conditions, are points upon which the many important practical bearings of this subject will depend.

\section{REPORT ON OPHTHALMIC CASES IN EGYPT,} I9I5

BY

Sir James W. Barrett,K.B.E.,C.M.G.,M.D.,M.S.,F.R.C.S.(Eng.) LIEUT.-COLONEL R.A.M.C.

THERE appeared in the August, 1917, number of this Journal valuable reports on the ophthalmic work done in Egypt in January, 1916, of the period Lieut.-Colonel Eason, C.M.G., Consulting Oculist, E.E.F., and from Major F. Lockhart Gibson, A.A.M.C., who was at Mudros in the latter part of 1915, and in Egypt for part of 1916. Both of these reports relate to the period when the work in Egypt was well organized, when appliances were freely obtainable, and when medical assistance was abundant.

1. I propose to complete the story by describing the ophthalmic work in Egypt during the period of improvisation, viz.: from the beginning of 1915 until November of the same year, when I was invalided out of Egypt. The actual work began about January 24, 1915, at the First Australian General Hospital, Heliopolis; where an ophthalmic and aural department was opened, to which patients speedily found their way. As there was no ophthalmic or aural surgeon available in Egypt at the time, I was appointed consulting oculist (and aurist) to the Force in Egypt, and the greater part of the work was done at Heliopolis. As the year passed by, oculists began to arrive in Egypt, and decentralization was gradually effected. The staff at Heliopolis usually consisted of two or three assistant surgeons, one medical student, and two nurses in the out-patient department, with the use of a ward of 100 beds. The assistant surgeons were mainly Captain Morlet, Captain Rosenfield, Captain Stevens, Captain MacLellan, and Captain Burke, A.A.M.C., and Mr. Cook and Mr. Wharton, medical students. The clinique was available, and utilized freely by British, and New Zealand, as well as Australian troops. In passing, it may be said that the work was very extensive and heavy, and that there was a considerable amount of operative work after the Dardanelles landing. 